\title{
Distal hereditary motor neuropathy type 7B with Dynactin 1 mutation
}

\author{
SUN HEE HWANG ${ }^{1 *}$, EUN JA KIM ${ }^{1 *}$, YOUNG BIN HONG ${ }^{2,3}$, JAESOON JOO $^{1}$, \\ SUNG MIN KIM ${ }^{4}$, SOO HYUN NAM ${ }^{4}$, HYUN DAE HONG ${ }^{4}$, SEUNG HYUN KIM ${ }^{5}$, KIWOOK OH ${ }^{5}$, \\ JEONG-GEUN LIM ${ }^{6}$, JEONG HEE CHO ${ }^{7}, \mathrm{KI} \mathrm{WHA} \mathrm{CHUNG}^{4}$ and BYUNG-OK CHOI ${ }^{1-3}$ \\ ${ }^{1}$ Department of Neurology, Samsung Medical Center, Sungkyunkwan University School of Medicine; \\ ${ }^{2}$ Stem Cell \& Regenerative Medicine Center and ${ }^{3}$ Neuroscience Center, Samsung Medical Center, Seoul 06351; \\ ${ }^{4}$ Department of Biological Sciences, Kongju National University, Gongju, South Chungcheong 32588; \\ ${ }^{5}$ Department of Neurology, Hanyang University College of Medicine, Seoul 04763; \\ ${ }^{6}$ Department of Neurology, Keimyung University College of Medicine, Daegu 42403; ${ }^{7}$ Department of Neurology, \\ National Health Insurance Corporation Ilsan Hospital, Goyang, Gyeonggi 10444, Republic of Korea
}

Received October 4, 2015; Accepted July 13, 2016

DOI: $10.3892 / \mathrm{mmr} .2016 .5664$

\begin{abstract}
Mutations in the Dynactin 1 (DCTN1) gene have been demonstrated to result in various neurodegenerative diseases, including distal hereditary motor neuropathy type 7B (dHMN7B), Perry syndrome, amyotrophic lateral sclerosis and amyotrophic lateral sclerosis-frontotemporal dementia. However, since the first dHMN7B patient with a DCTN1 mutation was described in 2003, to the best of our knowledge no further cases have been reported. In the present study, the DCTN1 p.G59S mutation was identified in two unrelated families from a total of 24 Korean families with dHMN, by whole exome sequencing. Codon 59 appears to be the mutational hot spot in the DCTN1 gene, as all described dHMN7B patients to date have harbored an identical p.G59S mutation. The families of the present study with the DCTN1 mutation had a milder disease with a later onset compared with the previously described patients. No affected family members exhibited facial muscle weakness or bulbar involvement. One family member demonstrated vocal cord palsy as the initial sign of
\end{abstract}

Correspondence to: Dr Byung-Ok Choi, Department of Neurology, Samsung Medical Center, Sungkyunkwan University School of Medicine, 81 Irwon-ro, Gangnam-gu, Seoul 06351, Republic of Korea

E-mail: bochoi77@hanmail.net

Professor Ki Wha Chung, Department of Biological Sciences, Kongju National University, 56 Gongjudaehak-ro, Gongju, South Chungcheong 32588, Republic of Korea

E-mail: kwchung@kongju.ac.kr

${ }^{*}$ Contributed equally

Key words: dynactin 1, distal hereditary motor neuropathy 7B, vocal cord, arytenoidectomy, frequency disease; however, in the other family hand muscle weakness was the first major symptom. No affected patients demonstrated sensory loss or upper motor neuron involvements. Although this is only the second report of dHMN7B resulting from a DCTN1 mutation, the frequency of the DCTN1 mutation was not low in the Korean population examined, and clinical heterogeneities were observed in patients with the DCTN1 mutation. Therefore, it may be beneficial to screen all dHMN patients for the DCTN1 mutation.

\section{Introduction}

Distal hereditary motor neuropathies (dHMN) are a genetically and clinically heterogeneous group of lower motor neuron diseases (1). dHMN type 7B (dHMN7B), which is caused by a mutation in the dynactin 1 (DCTN1) gene, is a late-onset disease characterized by respiratory difficulties due to bilateral vocal cord palsy, progressive facial weakness and muscle atrophy in the hands (2-4). In addition to dHMN7B, mutations in the DCTN1 gene are known to cause diverse neurodegenerative diseases, including dHMN7B, Perry syndrome (PS), amyotrophic lateral sclerosis (ALS) and ALS-frontotemporal dementia (ALS/FTD) (5-8). PS is an upper motor neuron disease characterized by Parkinsonism, psychiatric changes, weight loss and abnormal hypoventilation (8). ALS is characterized by the combined phenotypes of upper and lower motor neuron disorders (6). ALS/FTD presents with progressive changes in personality, behavior and language, with relative preservation of perception and memory, due to degeneration of the frontal and temporal lobes of brain (7).

The DCTN1 gene encodes the largest subunit of dynactin to provide commands for the synthesis of dynactin 1 (9-12). Dynactin 1 is important for the retrograde axonal transport of vesicles and organelles along microtubules $(1,13,14)$. It has been suggested that dysfunction of dynactin-mediated transport in the peripheral nerves may lead to motor neuron disease, and that mutation of the dynein-dynactin microtubule 
motor proteins leads to late-onset motor neuron disease in mice $(15,16)$.

The first dHMN7B patient with a DCTN1 mutation was described by Puls et al (5). To the best of our knowledge, no further cases have been reported. Thus, clinical information about dHMN7B resulting from a DCTN1 mutation has been limited to a single family. In the present study, 24 Korean dHMN families were screened using whole exome sequencing, which identified a DCTN1 mutation (p.G59S) in two unrelated families. In addition, the clinical and electrophysiological manifestations of the dHMN7B patients were described.

\section{Materials and methods}

Patients. The DCTN1 gene was sequenced in 62 individuals from 24 families with dHMN. Additionally, 300 Korean healthy controls with no clinical features or family history of peripheral neuropathies were recruited from the neurological department. Written informed consent was obtained from all individuals, according to the protocol approved by the Institutional Review Board for Sungkyunkwan University, Samsung Medical Center (Seoul, Korea).

Clinical assessments. Clinical evaluations were performed by neurologists and information on deceased family members was obtained from relatives. Clinical assessments included those for motor impairments, sensory loss, deep tendon reflexes and vocal cord palsy. Patient's age at onset was determined by questioning the patients regarding the first appearance of symptoms. Muscle strength of flexor and extensor muscles was measured manually using the standard Medical Research Council scale (17), physical disability was determined by the functional disability scale (18) and sensory impairment was assessed based on the level and severity of pain, temperature, vibration and position.

Electrophysiological study. Motor and sensory conduction velocities were measured on the median, ulnar, peroneal, tibial and sural nerves. Motor nerve conduction velocities (MNCVs) of the median and ulnar nerves were determined by stimulating at the elbow and wrist while recording compound muscle action potentials (CMAPs) over the abductor pollicis brevis and adductor digiti quinti, respectively. Similarly, the MNCVs of the peroneal and tibial nerves were determined by stimulating at the knee and ankle, while recording CMAPs over the extensor digitorum brevis and adductor hallucis, respectively. Sensory nerve conduction velocities were obtained over a finger-wrist segment from the median and ulnar nerves by orthodromic scoring, and in addition were recorded for the sural nerves. Sensory nerve action potential amplitudes were measured from positive to negative peaks.

DNA preparation and $17 p 12$ duplication analysis. DNA was purified from peripheral blood samples using a QIAamp Blood DNA Purification kit (Qiagen GmbH, Hilden, Germany). All patient samples were prescreened for $17 \mathrm{p} 12$ [peripheral myelin protein 22 (PMP22)] duplication and/or deletion, which is the most common genetic cause of peripheral neuropathies, using hexaplex microsatellite PCR analysis, as previously described (19).
Exome sequencing and identification of causative mutation. Whole exome sequencing (WES) was performed on two affected patients from two unrelated families. The exome was captured using the Human SeqCap EZ Human Exome Library kit (version 3.0; Roche NimbleGen, Inc., Madison, WI, USA), and subsequent sequencing was performed using a HiSeq 2500 Genome Analyzer (Illumina, Inc., San Diego, CA, USA). The University of California, Santa Cruz UCSC assembly hg19 (National Center for Biotechnology Information build 37.1; genome.ucsc.edu/) was used as the reference sequence with Burrows-Wheeler Aligner software version 07.11 (bio-bwa. sourceforge.net/). Using the Sequence Alignment/Map tools program (samtools.sourceforg.net/), variants were confirmed if the single nucleotide polymorphism (SNP) qualities were $\geq 20$. Functionally significant variants, including missense, nonsense, exonic indel and splicing site variants, were identified in $\sim 70$ peripheral neuropathy-associated genes. The sequencing variants were confirmed by the Sanger sequencing method using an ABI 3130xl genetic analyzer (Applied Biosystems; Thermo Fisher Scientific, Waltham, MA, USA) (20). A mutation was considered as causative when it was identified in affected individuals and not in the 300 healthy control samples.

In silico analysis. The conversion pattern for the protein amino acid sequence was performed in Molecular Evolutionary Genetics Analysis 5 software (version 5.05; www.megasoftware.net/) (21) and the effect of amino acid substitution on protein function was predicted using the online tools of Sorting Intolerant From Tolerant (SIFT; sift.jcvi.org/), Polymorphism Phenotyping version 2 (PolyPhen-2; genetics. bwh.harvard.edu/pph2/) and MUpro (mupro.proteomics.ics. uci.edu/). Genomic evolutionary rate profiling (GERP) score was determined using the GERP++ program (mendel.stanford.edu/SidowLab/downloads/gerp/index.html). The domain structure of DCTN1 protein was predicted using the Simple Modular Architecture Research Tool version 7 (smart.embl. $\mathrm{de} /)$.

\section{Results}

Clinical manifestations. The clinical presentations of the two families reported in the present study and the previously reported American family (5) are summarized in Table I. The average age of disease onset in the FC465 and FC654 families was $51.7 \pm 8.5$ years (mean \pm standard deviation; range, 43-60 years) and 53.8 \pm 5.4 years (range, 47-62 years), respectively, which indicated a later onset compared with the previously reported American family, in which the age at disease onset was $34.3 \pm 6.2$ years. In the two families described in the present study, no bulbar symptoms, including dysphagia, dysarthria and facial muscle weakness, were identified in affected members. The disease severity appeared milder in patients of the present study compared with the previously reported family (5). In all affected FC465 family members, stridor and shortness of breath were the first and predominant symptoms; however, in FC654 family members, hand muscle weakness occurred prior to vocal cord palsy. Members of the two families suffered from atrophy and weakness of the first dorsal interosseous and thenar muscles; however, the hypothenar muscle bulk and strength was relatively 
Table I. Clinical manifestations of dHMN7B patients with the dynactin 1 p.G59S mutation.

\begin{tabular}{lccc}
\hline & \multicolumn{2}{c}{ Korean dHMN7B families } & \\
\cline { 2 - 3 } Parameter & FC465 & FC654 & American dHMN7B family \\
\hline Number of patients & 3 & 4 & 9 \\
Inheritance & AD & AD & AD \\
Female ratio, $\%$ & 33.3 & 75.0 & 77.8 \\
Age at exam, years [mean \pm SD (range) $)$ & $56.3 \pm 7.5(52-65)$ & $60.3 \pm 4.1(55-61)$ & $51.9 \pm 8.3(39-64)$ \\
Age at onset, years [mean \pm SD (range)] & $51.7 \pm 8.5(43-60)$ & $53.8 \pm 5.4(47-62)$ & $34.3 \pm 6.2(23-44)$ \\
Frequent first symptom & Stridor & Hand weakness & Stridor \\
FDS & $2-3$ & $2-4$ & ND \\
Proximal limb muscle weakness & None to mild & None to mild & None to mild \\
Distal limb muscle weakness & Mild to moderate & Mild to severe & Mild to severe \\
Facial weakness & No & No & Yes \\
Sensory loss & No & No & No \\
Knee/ankle jerks & D, A & A & N, D, A \\
Vocal cord palsy & Yes & Yes & Yes \\
Arytenoidectomy & Yes & Yes & Yes \\
Dysphagia & No & No & Yes \\
Dyspnea & Yes & Yes & Yes \\
Foot drop & Bilateral & Bilateral & Bilateral \\
Wheelchair-bound & No & No & No \\
Tongue fasciculation & No & No & No \\
Frontotemporal dementia & No & No & No \\
Parkinsonism & No & No & No \\
Reference & The present study & The present study & Puls et al (2003) (5) \\
\hline
\end{tabular}

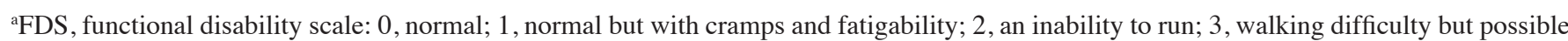

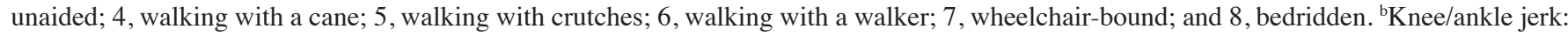
A, absent; D, decreased; and N, normal. dHMN7B, distal hereditary motor neuropathies type 7B; AD, autosomal dominant inheritance; SD, standard deviation; ND, not described.

preserved. All seven affected family members had undergone arytenoidectomy due to respiratory difficulty caused by bilateral vocal cord palsy. They had bilateral foot drop and steppage gait; however, no patient became wheelchair-bound. Patients from the two families exhibited no sensory loss or upper motor neuron involvement. Decreased or absent tendon reflexes were observed in the early stages of the disease, and pathologic reflexes were not identified. FC465 family members reported that the deceased father (I-1; Fig. 1A) and older brother (II-1; Fig. 1A) had bilateral vocal cord palsy and had undergone arytenoidectomy, and FC654 family members reported that the deceased mother (I-2; Fig. 1B) had bilateral vocal cord palsy and had undergone arytenoidectomy.

Pure hereditary motor neuropathy. The nerve conduction study data are summarized in Table II. The amplitude of the motor response from the thenar muscles was markedly reduced or absent in patients 1 [FC465 (II-6)] and 2 [FC654 (II-6)]; however, the amplitude of the response from the hypothenar muscles were minimally reduced. Patient 1 had reduced amplitudes of peroneal innervated foot muscles; however, these responses were reduced compared with the responses from the thenar muscles. Needle electromyography in the two patients was consistent with chronic denervation, and there were scattered fibrillations in distal limb muscles.

Identification of the DCTN1 mutation. Copy number variation on 17p12 (PMP22) was not observed; therefore WES was performed on the affected proband in each family. The mean total WES yield was $\sim 12.7 \mathrm{Gbp} / \mathrm{sample}$ with a $94.37 \%$ coverage rate ( $\geq 10 \mathrm{X}$ read depth) for the target regions. The average number of observed coding variants was $\sim 24,765$ SNPs including insertion/deletion variants.

By filtering of the functionally significant variants in $>70$ peripheral neuropathy-associated genes, the missense mutation in the DCTN1 gene was identified in two dHMN families: c.175G>A (p.G59S) in FC465 and FC654 families. This mutation was confirmed by the Sanger's DNA sequencing method (Fig. 2A). The p.G59S was located in the cytoskeleton-associated protein glycine-rich domain. This mutation was not observed in any of the 300 healthy controls, and it was not reported in the dbSNP144 database (www.ncbi.nlm.nih.gov), the 1000 Genomes project database (www.1000genomes.org/) or the Exome Variant Server (evs.gs.washington.edu/EVS/). Conservation analysis revealed that the vicinity of mutation site was conserved among the species assessed (Fig. 2B). 
A
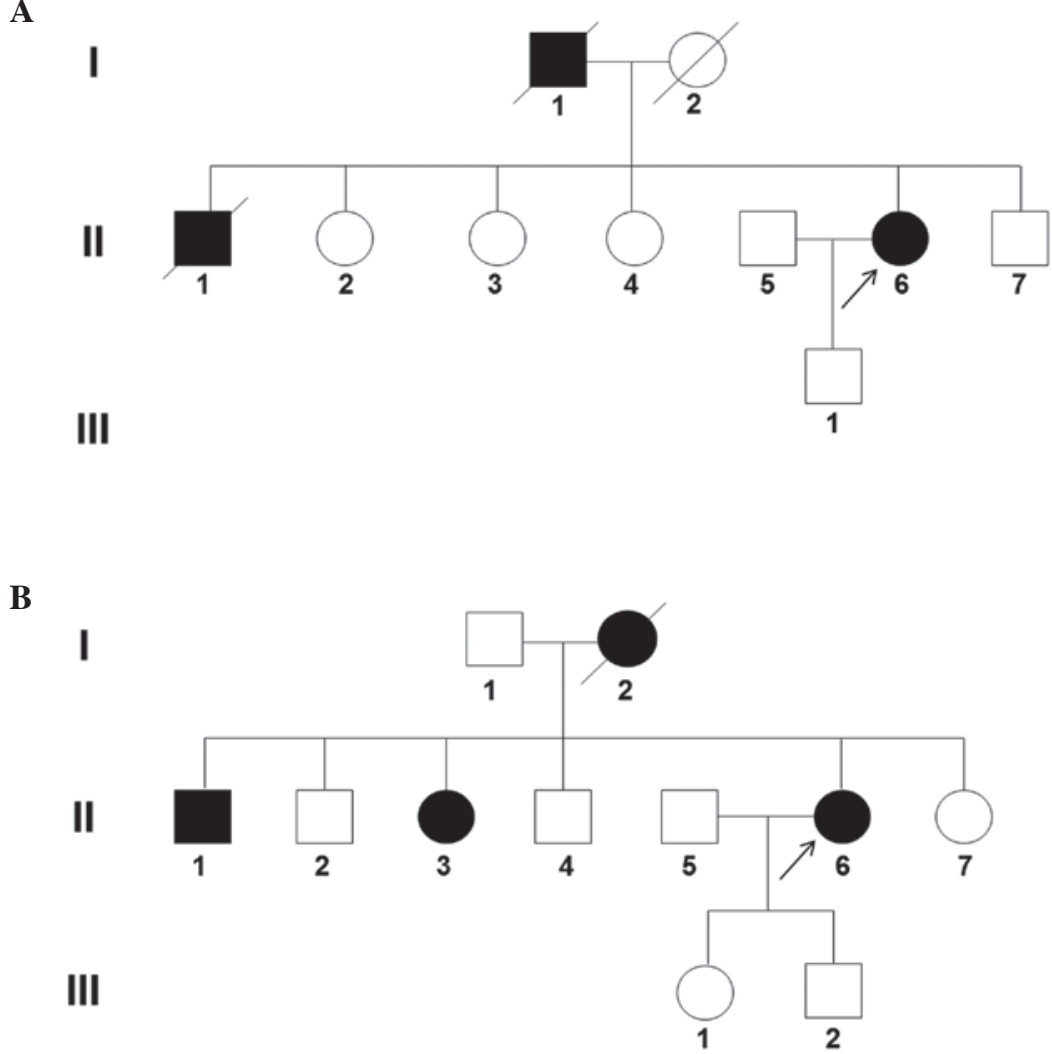

Figure 1. Pedigree analyses of two distal hereditary motor neuropathies type 7B families. (A) Pedigree analysis of the FC654 family. (B) Pedigree analysis of the FC465 family. Open and filled symbols represent unaffected and affected individuals, respectively. Arrows indicate the probands.

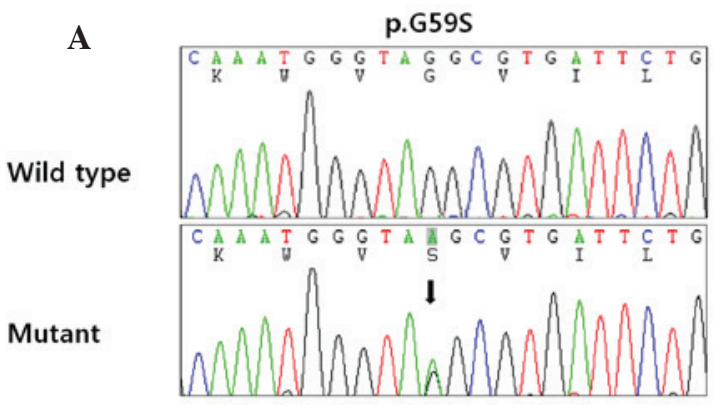

$\mathbf{B}$

p.G59S

\begin{tabular}{|l|l|l|l|l|l|l|l|l|l|l|l|l|l|l|l|}
\hline Homo sapiens & F & A & $\mathbf{T}$ & $\mathbf{G}$ & $\mathbf{K}$ & $\mathbf{W}$ & $\mathbf{V}$ & $\mathbf{G}$ & $\mathbf{V}$ & $\mathbf{I}$ & $\mathbf{L}$ & $\mathbf{D}$ & $\mathbf{E}$ & $\mathbf{A}$ & $\mathbf{K}$ \\
\hline Mus musculus & F & $\mathbf{A}$ & $\mathbf{T}$ & $\mathbf{G}$ & $\mathbf{K}$ & $\mathbf{W}$ & $\mathbf{V}$ & $\mathbf{G}$ & $\mathbf{V}$ & $\mathbf{I}$ & $\mathbf{L}$ & $\mathbf{D}$ & $\mathbf{E}$ & $\mathbf{A}$ & $\mathbf{K}$ \\
\hline Bos taurus & F & $\mathbf{A}$ & $\mathbf{T}$ & $\mathbf{G}$ & $\mathbf{K}$ & $\mathbf{W}$ & $\mathbf{V}$ & $\mathbf{G}$ & $\mathbf{V}$ & $\mathbf{I}$ & $\mathbf{L}$ & $\mathbf{D}$ & $\mathbf{E}$ & $\mathbf{A}$ & $\mathbf{K}$ \\
\hline Gallus gallus & X & $\mathbf{A}$ & $\mathbf{T}$ & $\mathbf{G}$ & $\mathbf{K}$ & $\mathbf{W}$ & $\mathbf{V}$ & $\mathbf{G}$ & $\mathbf{V}$ & $\mathbf{I}$ & $\mathbf{L}$ & $\mathbf{D}$ & $\mathbf{E}$ & $\mathbf{A}$ & $\mathbf{K}$ \\
\hline Xenopus tropicalis & F & $\mathbf{A}$ & $\mathbf{T}$ & $\mathbf{G}$ & $\mathbf{K}$ & $\mathbf{W}$ & $\mathbf{V}$ & $\mathbf{G}$ & $\mathbf{V}$ & $\mathbf{I}$ & $\mathbf{L}$ & $\mathbf{D}$ & $\mathbf{D}$ & $\mathbf{S}$ & $\mathbf{K}$ \\
\hline Poecilia reticulata & F & $\mathbf{A}$ & $\mathbf{S}$ & $\mathbf{G}$ & $\mathbf{K}$ & $\mathbf{W}$ & $\mathbf{V}$ & $\mathbf{G}$ & $\mathbf{V}$ & $\mathbf{I}$ & $\mathbf{L}$ & $\mathbf{D}$ & $\mathbf{E}$ & $\mathbf{P}$ & $\mathbf{K}$ \\
\hline Bactrocera dorsalis & F & $\mathbf{A}$ & $\mathbf{V}$ & $\mathbf{G}$ & $\mathbf{K}$ & $\mathbf{W}$ & $\mathbf{V}$ & $\mathbf{G}$ & $\mathbf{V}$ & $\mathbf{V}$ & $\mathbf{L}$ & $\mathbf{D}$ & $\mathbf{E}$ & $\mathbf{P}$ & $\mathbf{K}$ \\
\hline
\end{tabular}

C

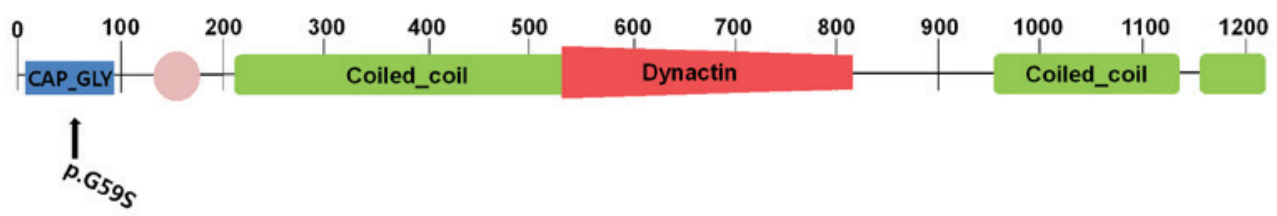

Figure 2. Sequencing chromatograms and conservation analysis of DCTN1 mutations. (A) Sequencing chromatograms of the mutation. Mutation site is indicated by a vertical arrow. (B) Conservation of amino acid sequences in the mutation site. Multiple protein sequence alignment revealed conservation of the p.G59S mutation site among various animal species (Homo sapiens, NM_004082.4; Mus musculus, NP_001185796.1; Bos taurus, NP_001092404.1; Gallus gallus, NP 001026538.1; Xenopus tropicalis, XP 002936330.2; Poecilia reticulata, XP 008397058.1; and Bactrocera dorsalis, XP 011199932.1). (C) DCTN1 protein structure. Domains were drawn using the Simple Modular Architecture Research Tool (smart.embl.de/). The identified missense mutation is indicated in the CAP_GLY domain. DCTN1, dynactin 1; CAP_GLY, cytoskeleton-associated proteins_glycine-rich domain.

The p.G59S mutation has been previously reported to be the underlying cause of dHMN7B (5). Various causative mutations for other neurodegenerative diseases were located in the coiled-coil domain (Fig. 2C) (7,8). The GERP score 
Table II. Electrophysiology in patients with the dynactin 1 p.G59S mutation.

A, Motor nerve conduction velocities: Median motor nerve

\begin{tabular}{|c|c|c|c|c|c|}
\hline \multirow[b]{2}{*}{ Parameter } & \multicolumn{2}{|c|}{ Patient FC465 (II-6) } & \multicolumn{2}{|c|}{ Patient FC654 (II-6) } & \multirow[b]{2}{*}{ Normal value } \\
\hline & Right side & Left side & Right side & Left side & \\
\hline $\mathrm{TL}, \mathrm{ms}$ & $\mathrm{A}$ & 3.8 & A & A & $<3.9$ \\
\hline CMAP, mV & A & 1.2 & A & $\mathrm{A}$ & $>6.0$ \\
\hline $\mathrm{MNCV}, \mathrm{m} / \mathrm{s}$ & A & 35.1 & A & $\mathrm{A}$ & $>50.5$ \\
\hline
\end{tabular}

B, Motor nerve conduction velocities: Ulnar motor nerve

\begin{tabular}{lcrlrrr}
\hline & \multicolumn{2}{c}{ Patient FC465 (II-6) } & & \multicolumn{2}{c}{ Patient FC654 (II-6) } \\
\cline { 2 - 3 } Parameter & Right side & Left side & & Right side & Left side & Normal value \\
\hline TL, ms & 2.2 & 2.6 & & 2.5 & 3.0 & $<3.0$ \\
CMAP, $\mathrm{mV}$ & 8.9 & 12.5 & & 7.5 & 3.8 & $>8.0$ \\
MNCV, $\mathrm{m} / \mathrm{s}$ & 54.5 & 55.6 & 52.2 & 64.2 & $>51.1$ \\
\hline
\end{tabular}

C, Motor nerve conduction velocities: Peroneal nerve

\begin{tabular}{lcccccc}
\hline & \multicolumn{2}{c}{ Patient FC465 (II-6) } & & \multicolumn{2}{c}{ Patient FC654 (II-6) } \\
\cline { 2 - 3 } Parameter & Right side & Left side & & Right side & Left side & Normal value \\
\hline TL, ms & 3.7 & 3.8 & & 4.0 & 3.8 & $<.3$ \\
CMAP, $\mathrm{mV}$ & 4.7 & 4.4 & & 0.6 & 0.1 & $>1.6$ \\
MNCV, $\mathrm{m} / \mathrm{s}$ & 43.2 & 43.9 & 48.1 & 43.7 & $>41.2$ \\
\hline
\end{tabular}

D, Motor nerve conduction velocities: Tibial nerve

\begin{tabular}{lcrlrr}
\hline & \multicolumn{2}{c}{ Patient FC465 (II-6) } & & \multicolumn{2}{c}{ Patient FC654 (II-6) } \\
\cline { 2 - 3 } Parameter & Right side & Left side & & Right side & Left side \\
\hline TL, ms & 4.0 & 4.3 & 3.9 & 3.3 & Normal value \\
CMAP, $\mathrm{mV}$ & 8.5 & 10.5 & 11.2 & 10.8 & $>5.4$ \\
MNCV, $\mathrm{m} / \mathrm{s}$ & 42.7 & 41.9 & 48.5 & 47.8 & $>41.1$ \\
\hline
\end{tabular}

E, Sensory nerve conduction velocities: Median sensory nerve

\begin{tabular}{lccccc}
\hline & \multicolumn{2}{c}{ Patient FC465 (II-6) } & & \multicolumn{2}{c}{ Patient FC654 (II-6) } \\
\cline { 2 - 3 } Parameter & Right side & Left side & & Right side & Left side \\
\hline SNAP, $\mu \mathrm{V}$ & 11.9 & 29.2 & 55.6 & 43.4 & Normal value \\
SNCV, $\mathrm{m} / \mathrm{s}$ & 41.7 & 40.0 & 47.1 & 46.0 & $>8.8$ \\
\hline
\end{tabular}

F, Sensory nerve conduction velocities: Ulnar sensory nerve

\begin{tabular}{lccccc}
\hline & \multicolumn{2}{c}{ Patient FC465 (II-6) } & & \multicolumn{2}{c}{ Patient FC654 (II-6) } \\
\cline { 2 - 3 } Parameter & Right side & Left side & & Right side & Left side \\
\hline SNAP, $\mu \mathrm{V}$ & 8.9 & 22.3 & 35.6 & 12.8 & Normal value \\
SNCV, $\mathrm{m} / \mathrm{s}$ & 44.6 & 41.7 & 44.4 & 40.6 & $>37.9$ \\
\hline
\end{tabular}


Table II. Continued.

G, Sensory nerve conduction velocities: Sural nerve

\begin{tabular}{|c|c|c|c|c|c|}
\hline \multirow[b]{2}{*}{ Parameter } & \multicolumn{2}{|c|}{ Patient FC465 (II-6) } & \multicolumn{2}{|c|}{ Patient FC654 (II-6) } & \multirow[b]{2}{*}{ Normal value } \\
\hline & Right side & Left side & Right side & Left side & \\
\hline SNAP, $\mu \mathrm{V}$ & 30.7 & 24.7 & 22.4 & 24.0 & $>6.0$ \\
\hline $\mathrm{SNCV}, \mathrm{m} / \mathrm{s}$ & 34.1 & 35.6 & 44.7 & 47.2 & $>32.1$ \\
\hline
\end{tabular}

Patient FC465 was examined at 51 years of age, and patient FC654 was examined at 61 years of age. A, absent potentials; TL, terminal latency; CMAP, compound muscle action potential; MNCV, motor nerve conduction velocity; SNAP, sensory nerve action potential; SNCV, sensory nerve conduction velocity.

Table III. In silico analysis of dynactin 1 mutations.

\begin{tabular}{lrl}
\hline $\begin{array}{l}\text { In silico } \\
\text { analysis }\end{array}$ & $\begin{array}{c}\text { c.175G }>\mathrm{A} \\
(\mathrm{p} . \mathrm{G} 59 \mathrm{~S})\end{array}$ & \multicolumn{1}{c}{ Prediction } \\
\hline SIFT & $0.000^{\mathrm{a}}$ & Affection of protein function \\
PolyPhen-2 & $1.000^{\mathrm{a}}$ & Damaging \\
MUUpro & $-0.880^{\mathrm{a}}$ & Decreased of structure stability \\
\hline
\end{tabular}

aPathogenic prediction (SIFT score $\leq 0.05$, PolyPhen 2 score $\sim 1$, and MUpro score $<0$ indicate a prediction of pathogenicity). SIFT, Sorting Intolerant From Tolerant (sift.jcvi.org/); PolyPhen-2, Polymorphism Phenotyping version 2 (genetics.bwh.harvard.edu/pph2/); MUpro (mupro.proteomics.ics.uci.edu/).

of 5.14 was considerably high in the two mutated nucleotide sequences, and in silico analyses using SIFT, PolyPhen-2 and MUpro predicted the affected function of the mutant protein (Table III). Although other non-synonymous variants were identified in the peripheral neuropathy-associated genes, with the exception of the DCTN1 mutations, no other variants were considered as the underlying cause of $\mathrm{dHMN}$, as they were identified in healthy controls.

\section{Discussion}

In the present study, a p.G59S mutation was identified as the underlying cause of dHMN7B in two families, and their detailed clinical features were characterized. This is the second description of dHMN7B resulting from a DCTN1 mutation, following the initial report in 2003 (5). Codon 59 appears to be the mutational hot spot in the DCTN1 gene, as all described dHMN7B patients to date have harbored identical p.G59S mutations.

The families with the DCTN1 mutation reported in the present study had similar clinical features to the previously described family. All patients underwent an arytenoidectomy to provide an adequate airway and hand muscle weakness affected the thenar more than the hypothenar muscles. All patients had steppage gait, but none became wheelchair-bound. However, clinical heterogeneities were observed between the present and previously reported patients. The age of onset in the FC465 and FC654 families was later compared with the previously reported American family. No affected family members of the present study exhibited any bulbar symptoms, including facial weakness, dysphagia and dysarthria. The severity of the disease manifestations was milder compared with that in the previously reported family. In addition, interfamilial differences were observed. In affected FC465 members, respiratory difficulty during exercise was the initial and predominant symptom; however, in affected FC654 members, hand muscle weakness was the first dominant symptom. Thus, diverse clinical features of an identical DCTN1 mutation were observed; this may be useful for the future diagnosis of dHMN7B patients with a DCTN1 mutation.

The phenotype of the disease in the patients of the present study differs from other diseases associated with DCTN1 mutations, including ALS, ALS/FTD and PS, in important aspects. The most significant difference is the absence of lower motor neuron signs in the other diseases. All patients of the present study exhibited decreased or absent deep tendon reflexes; however, patients with ALS, ALS/FTD or PS exhibited normal or increased deep tendon reflexes. Furthermore, the pathognomonic symptoms of disease in the patients of the present study did not tend to overlap with ALS, ALS/FTD and PS (Table I).

The frequency of dHMN7B resulting from a DCTN1 mutation appears to be extremely low. To the best of our knowledge, no cases have been reported since the first report of dHMN7B family with p.G59S DCTN1 mutation (5). However, the present study identified the p.G59S mutation of the DCTN1 gene in two Korean dHMN families from 24 unrelated families $(8.3 \%)$, which is not extremely rare. It may be that this mutation is relatively common in Asia and rare in the west. In addition, DCTN1 mutations have been demonstrated to result in upper motor neuron diseases, including PS, ALS and ALS/FTD. To date, mutations of the DCTN1 gene have not generally been considered to cause lower motor neuron diseases, due to the lack of evidence supporting the initial report in $\mathrm{dHMN7B}$. In conclusion, the results of the present study suggested that the DCTN1 mutation may not be extremely rare, particularly in the Korean population, and therefore screening for the DCTN1 gene must be considered for all dHMN patients. 


\section{Acknowledgements}

The authors would like to thank the patients and their families for their participation. The present study was supported by the Korean Health Technology R\&D Project, Ministry of Health \& Welfare (grant nos. HI12C0135 and HI14C3484) and by the National Research Foundation of Korea grants funded by the Ministry of Science, ICT and Future Planning (grant no. NRF-2014R1A2A2A01004240).

\section{References}

1. Rossor AM, Kalmar B, Greensmith L and Reilly MM: The distal hereditary motor neuropathies. J Neurol Neurosurg Psychiatry 83: 6-14, 2012.

2. Puls I, Oh SJ, Sumner CJ, Wallace KE, Floeter MK, Mann EA, Kennedy WR, Wendelschafer-Crabb G, Vortmeyer A, Powers R, et al: Distal spinal and bulbar muscular atrophy caused by dynactin mutation. Ann Neurol 57: 687-694, 2005.

3.2nd Workshop of the European CMT Consortium: 53rd ENMC International Workshop on Classification and Diagnostic Guidelines for Charcot-Marie-Tooth Type 2 (CMT2-HMSN II) and Distal Hereditary Motor Neuropathy (distal HMN-Spinal CMT) 26-28 September 1997, Naarden, The Netherlands. Neuromuscul Disord 8: 426-431, 1998.

4. Harding AE and Thomas PK: Genetic aspects of hereditary motor and sensory neuropathy (types I and II). J Med Genet 17: 329-336, 1980.

5. Puls I, Jonnakuty C, LaMonte BH, Holzbaur EL, Tokito M, Mann E, Floeter MK, Bidus K, Drayna D, Oh SJ, et al: Mutant dynactin in motor neuron disease. Nat Genet 33: 455-456, 2003.

6. Münch C, Sedlmeier R, Meyer T, Homberg V, Sperfeld AD, Kurt A, Prudlo J, Peraus G, Hanemann CO, Stumm G and Ludolph AC: Point mutations of the p150 subunit of dynactin (DCTN1) gene in ALS. Neurology 63: 724-726, 2004.

7. Münch C, Rosenbohm A, Sperfeld AD, Uttner I, Reske S, Krause BJ, Sedlmeier R, Meyer T, Hanemann CO, Stumm G and Ludolph AC: Heterozygous R $1101 \mathrm{~K}$ mutation of the DCTN1 gene in a family with ALS and FTD. Ann Neurol 58: 777-780, 2005.

8. Farrer MJ, Hulihan MM, Kachergus JM, Dächsel JC, Stoessl AJ, Grantier LL, Calne S, Calne DB, Lechevalier B Chapon F, et al: DCTN1 mutations in Perry syndrome. Nat Genet 41: 163-165, 2009.
9. Irobi J, Dierick I, Jordanova A, Claeys KG, De Jonghe P and Timmerman V: Unraveling the genetics of distal hereditary motor neuronopathies. Neuromolecular Med 8: 131-146, 2006.

10. Holzbaur EL and Vallee RB: DYNEINS: Molecular structure and cellular function. Annu Rev Cell Biol 10: 339-372, 1994.

11. Irobi J, De Jonghe P and Timmerman V: Molecular genetics of distal hereditary motor neuropathies. Hum Mol Genet 13: R195-R202, 2004.

12. Moore JK, Sept D and Cooper JA: Neurodegeneration mutations in dynactin impair dynein-dependent nuclear migration. Proc Natl Acad Sci USA 106: 5147-5152, 2009.

13. Levy JR, Sumner CJ, Caviston JP, Tokito MK, Ranganathan S, Ligon LA, Wallace KE, LaMonte BH, Harmison GG, Puls I, et al: A motor neuron disease-associated mutation in p150Glued perturbs dynactin function and induces protein aggregation. J Cell Biol 172: 733-745, 2006.

14. Vaughan KT and Vallee RB: Cytoplasmic dynein binds dynactin through a direct interaction between the intermediate chains and p150Glued. J Cell Biol 131: 1507-1516, 1995.

15. LaMonte BH, Wallace KE, Holloway BA, Shelly SS, Ascaño J, Tokito M, Van Winkle T, Howland DS and Holzbaur EL: Disruption of dynein/dynactin inhibits axonal transport in motor neurons causing late-onset progressive degeneration. Neuron 34: 715-727, 2002.

16. Hafezparast M, Klocke R, Ruhrberg C, Marquardt A, Ahmad-Annuar A, Bowen S, Lalli G, Witherden AS, Hummerich H, Nicholson S, et al: Mutations in dynein link motor neuron degeneration to defects in retrograde transport. Science 300: 808-812, 2003.

17. Paternostro-Sluga T, Grim-Stieger M, Posch M, Schuhfried O, Vacariu G, Mittermaier C, Bittner C and Fialka-Moser V: Reliability and validity of the Medical Research Council (MRC) scale and a modified scale for testing muscle strength in patients with radial palsy. J Rehabil Med 40: 665-671, 2008.

18. Birouk N, Gouider R, Le Guern E, Gugenheim M, Tardieu S, Maisonobe T, Le Forestier N, Agid Y, Brice A and Bouche P: Charcot-Marie-Tooth disease type 1A with 17p11.2 duplication. Clinical and electrophysiological phenotype study and factors influencing disease severity in 119 cases. Brain 120: 813-823, 1997.

19. Choi BO, Kim J, Lee KL, Yu JS, Hwang JH and Chung KW: Rapid diagnosis of CMT1A duplications and HNPP deletions by multiplex microsatellite PCR. Mol Cells 23: 39-48, 2007.

20. Sanger F, Nicklen S and Coulson AR: DNA sequencing with chain-terminating inhibitors. Proc Natl Acad Sci USA 74: 5463-5467, 1977.

21. Tamura K, Peterson D, Peterson N, Stecher G, Nei M and Kumar S: MEGA5: Molecular evolutionary genetics analysis using maximum likelihood, evolutionary distance, and maximum parsimony methods. Mol Biol Evol 28: 2731-2739, 2011. 